\title{
Corrigendum: Gs-coupled GPCR signalling in AgRP neurons triggers sustained increase in food intake
}

Ken-ichiro Nakajima, Zhenzhong Cui, Chia Li, Jaroslawna Meister, Yinghong Cui, Ou Fu, Adam S. Smith, Shalini Jain, Bradford B. Lowell, Michael J. Krashes \& Jürgen Wess

Nature Communications 7:10268 doi: 10.1038/ncomms10268 (2016); Published 8 Jan 2016; Updated 3 Mar 2016

The financial support for this article was not fully acknowledged. The Acknowledgements should have included the following:

This work was supported by the intramural research program of the NIMH (ZIA-MH-002498-24). 